\title{
Counting nonsingular matrices with primitive row vectors *
}

\author{
Samuel Holmin
}

June 20, 2018

\begin{abstract}
We give an asymptotic expression for the number of nonsingular integer $n \times n$-matrices with primitive row vectors, determinant $k$, and Euclidean matrix norm less than $T$, as $T \rightarrow \infty$.

We also investigate the density of matrices with primitive rows in the space of matrices with determinant $k$, and determine its asymptotics for large $k$.
\end{abstract}

\section{Introduction}

An integer vector $v \in \mathbb{Z}^{n}$ is primitive if it cannot be written as an integer multiple $m \neq 1$ of some other integer vector $w \in \mathbb{Z}^{n}$. Let $A$ be an integer $n \times n$-matrix with nonzero determinant $k$ and primitive row vectors. We ask how many such matrices $A$ there are of Euclidean norm at most $T$, that is, $\|A\| \leq T$, where $\|A\|:=\sqrt{\sum a_{i j}^{2}}=\sqrt{\operatorname{tr}\left(A^{t} A\right)}$. Let $N_{n, k}^{\prime}(T)$ be this number (the prime in the notation denotes the primitivity of the rows), and let $N_{n, k}(T)$ be the corresponding counting function for matrices with not necessarily primitive row vectors. We will determine the asymptotic behavior of $N_{n, k}^{\prime}(T)$ for large $T$, and investigate the density $D_{n}(k):=\lim _{T \rightarrow \infty} N_{n, k}^{\prime}(T) / N_{n, k}(T)$ of matrices with primitive vectors in the space of matrices with nonzero determinant $k$. Since $N_{n, k}^{\prime}$ and $N_{n, k}$ do not depend on the sign of $k$, we will without loss of generality assume that $k>0$.

Let $M_{n, k}$ be the set of integer $n \times n$-matrices with determinant $k$. Then $N_{n, k}(T)=$ $\left|B_{T} \cap M_{n, k}\right|$, where $B_{T}$ is the (closed) ball of radius $T$ centered at the origin in the space $M_{n}(\mathbb{R})$ of real $n \times n$-matrices equipped with the Euclidean norm. Throughout, we will assume that $n \geq 2$ and $k>0$ unless stated otherwise.

Duke, Rudnick and Sarnak [DRS93] found that the asymptotic behavior of $N_{n, k}$ is given by

$$
N_{n, k}(T)=c_{n, k} T^{n(n-1)}+O_{\varepsilon}\left(T^{n(n-1)-1 /(n+1)+\varepsilon}\right),
$$

\footnotetext{
*The author was partially supported by the Swedish Research Council.
} 
as $T \rightarrow \infty$, for a certain constant $c_{n, k}$ and all $\varepsilon>0$, where the error term can be improved to $O\left(T^{4 / 3}\right)$ for $n=2$. The corresponding case for singular matrices was later investigated by Katznelson, who proved in [Kat93 that

$$
N_{n, 0}(T)=c_{n, 0} T^{n(n-1)} \log T+O\left(T^{n(n-1)}\right) .
$$

See the next page for the constants $c_{n, k}$ and $c_{n, 0}$.

Let $M_{n, k}^{\prime}$ be the set of matrices in $M_{n, k}$ with primitive row vectors. Then $N_{n, k}^{\prime}(T)=$ $\left|B_{T} \cap M_{n, k}^{\prime}\right|$. Wigman Wig05 determined the asymptotic behavior of the counting function $\left|G_{T} \cap M_{n, 0}^{\prime}\right|$, where $G_{T}$ is a ball of radius $T$ in $M_{n}(\mathbb{R})$, under a slightly different norm than ours. The results can be transferred to our setting, whereby we have

$$
\begin{aligned}
& N_{n, 0}^{\prime}(T)=c_{n, 0}^{\prime} T^{n(n-1)} \log T+O\left(T^{n(n-1)}\right), \quad n \geq 4, \\
& N_{3,0}^{\prime}(T)=c_{3,0}^{\prime} T^{3(3-1)} \log T+O\left(T^{3(3-1)} \log \log T\right), \\
& N_{2,0}^{\prime}(T)=c_{2,0}^{\prime} T^{2(2-1)}+O(T) .
\end{aligned}
$$

The case $n=2$ above is equivalent to the primitive circle problem, which asks how many primitive vectors there are of length at most $T$ in $\mathbb{Z}^{2}$ given any (large) $T$.

The main result in our paper is the following asymptotic expression for the number of nonsingular matrices with primitive row vectors and fixed determinant.

Theorem 1. Let $k \neq 0$. Then

$$
N_{n, k}^{\prime}(T)=c_{n, k}^{\prime} T^{n(n-1)}+O_{\varepsilon}\left(T^{n(n-1)-1 /(2 n)+\varepsilon}\right),
$$

as $T \rightarrow \infty$ for a certain constant $c_{n, k}^{\prime}$ and all $\varepsilon>0$.

Section 3 is dedicated to the proof of this theorem.

The constant in Theorem 1 can be written as

$$
c_{n, k}^{\prime}=\frac{C_{1}}{|k|^{n-1}} \sum_{d_{1} \cdots d_{n}=|k|} \prod_{i=1}^{n} \sum_{g \mid d_{i}} \mu(g)\left(\frac{d_{i}}{g}\right)^{i-1},
$$

for $k \neq 0$, which may be compared to the constants obtained from [DRS93, [Kat93] and Wig05, namely

$$
\begin{aligned}
& c_{n, k}=\frac{C_{1}}{|k|^{n-1}} \sum_{d_{1} \cdots d_{n}=|k|} \prod_{i=1}^{n} d_{i}^{i-1} \\
& c_{n, 0}=C_{0} \frac{n-1}{\zeta(n)} \\
& c_{n, 0}^{\prime}= \begin{cases}C_{0} \frac{n-1}{\zeta(n-1)^{n} \zeta(n)} & (n \geq 3) \\
\frac{\pi T^{2}}{\zeta(2)} & (n=2)\end{cases}
\end{aligned}
$$


where $\zeta$ is the Riemann zeta function, $\mu$ is the Möbius function, and $C_{0}$ and $C_{1}$ are constants defined as follows (these depend on $n$, but we will always regard $n$ as fixed). Let $\nu$ be the normalized Haar measure on $\mathrm{SL}_{n}(\mathbb{R})$. The measure $w$ below is obtained by averaging the $n(n-1)$-dimensional volume of $E \cap A_{u}$ over all classes $A_{u}:=\{A \in$ $\left.M_{n}(\mathbb{R}): A u=0\right\}$ for nonzero $u \in \mathbb{R}^{n}$. In Appendix $\mathbb{C}$ we give a precise definition of $w$ and calculate $w\left(B_{1}\right)$.

Write $V_{n}$ for the volume of the unit ball in $\mathbb{R}^{n}$ and $S_{n-1}$ for the surface area of the $(n-1)$-dimensional unit sphere in $\mathbb{R}^{n}$. Then

$$
\begin{aligned}
& C_{0}:=w\left(B_{1}\right)=\frac{V_{n(n-1)} S_{n-1}}{2}=\frac{\pi^{n^{2} / 2}}{\Gamma\left(\frac{n}{2}\right) \Gamma\left(\frac{n(n-1)}{2}+1\right)}, \\
& C_{1}:=\lim _{T \rightarrow \infty} \frac{\nu\left(B_{T} \cap \mathrm{SL}_{n}(\mathbb{R})\right)}{T^{n(n-1)}}=\frac{V_{n(n-1)} S_{n-1}}{2 \zeta(2) \cdots \zeta(n)}=\frac{C_{0}}{\zeta(2) \cdots \zeta(n)} .
\end{aligned}
$$

\subsection{Density}

It will be interesting to compare the growth of $N_{n, k}^{\prime}$ to that of $N_{n, k}$. We define the density of matrices with primitive rows in the space $M_{n, k}$ to be

$$
D_{n}(k):=\lim _{T \rightarrow \infty} \frac{N_{n, k}^{\prime}(T)}{N_{n, k}(T)}=\frac{c_{n, k}^{\prime}}{c_{n, k}} .
$$

The asymptotics of $N_{n, 0}$ and $N_{n, 0}^{\prime}$ are known from Kat93 and Wig05, and taking their ratio, we see that

$$
D_{n}(0)=\frac{1}{\zeta(n-1)^{n}}
$$

for $n \geq 3$. We will be interested in the value of $D_{n}(k)$ for large $n$ and large $k$. The limit of $D_{n}(k)$ as $k \rightarrow \infty$ does not exist, but it does exist for particular sequences of $k$.

We say that a sequence of integers is totally divisible if its terms are eventually divisible by all positive integers smaller than $m$, for any $m$. We say that a sequence of integers is rough if its terms eventually have no divisors smaller than $m$ (except for 1 ), for any $m$. An equivalent formulation is that a sequence $\left(k_{1}, k_{2}, \ldots\right)$ is totally divisible if and only if $\left|k_{i}\right|_{p} \rightarrow 0$ as $i \rightarrow \infty$ for all primes $p$, and $\left(k_{1}, k_{2}, \ldots\right)$ is rough if and only if $\left|k_{i}\right|_{p} \rightarrow 1$ as $i \rightarrow \infty$ for all primes $p$, where $|m|_{p}$ denotes the $p$-adic norm of $m$.

We state our main results about the density $D_{n}$. We prove these in section 4 ,

Theorem 2. Let $n \geq 3$ be fixed. Then $D_{n}$ is a multiplicative function, and $D_{n}\left(p^{m}\right)$ is strictly decreasing as a function of $m$ for any prime $p$. We have

$$
\frac{1}{\zeta(n-1)^{n}}=D_{n}(0)<D_{n}(k)<D_{n}(1)=1
$$

for all $k \neq 0,1$. Now let $k_{1}, k_{2}, \ldots$ be a sequence of integers. Then

$$
D_{n}\left(k_{i}\right) \rightarrow 1
$$


if and only if $\left(k_{1}, k_{2}, \ldots\right)$ is a rough sequence, and

$$
D_{n}\left(k_{i}\right) \rightarrow \frac{1}{\zeta(n-1)^{n}}
$$

if and only if $\left(k_{1}, k_{2}, \ldots\right)$ is a totally divisible sequence. Moreover, $D_{n}(k) \rightarrow 1$ uniformly as $n \rightarrow \infty$.

Remark 3. Given an integer sequence $k_{1}, k_{2}, \ldots$, write $k_{i}= \pm \prod_{p} p^{m_{p}(i)}$ for the prime decomposition of $k_{i}$ for nonzero $k_{i}$, and otherwise formally define $m_{p}(i)=\infty$ for all $p$ if $k_{i}$ is zero. For $n \geq 3$, it follows from Theorem 2 that the $\operatorname{limit}_{\lim _{i \rightarrow \infty}} D_{n}\left(k_{i}\right)$ exists and is equal to $\prod_{p} \lim _{i \rightarrow \infty} D_{n}\left(p^{m_{p}(i)}\right)$ where the product extends over all primes $p$, whenever every sequence of prime exponents $\left(m_{p}(1), m_{p}(2), \ldots\right)$ is either eventually constant or tends to $\infty$.

We prove Theorem 2 for nonzero $k_{i}$, but it is interesting that this formulation holds for $k=0$ also. The case of $k=0$ was proved by Wigman Wig05, where he found that $D_{n}(0)$ equals $1 / \zeta(n-1)^{n}$. We remark that Theorem 2 implies that

$$
D_{n}\left(k_{i}\right) \rightarrow D_{n}(0)
$$

if and only if $\left(k_{1}, k_{2}, \ldots\right)$ is totally divisible, for any fixed $n \geq 3$.

For completeness, let us state what happens in the rather different case $n=2$.

Proposition 4. Let $n=2$. Then $D_{n}$ is a multiplicative function, and $D_{n}\left(p^{m}\right)$ is strictly decreasing as a function of $m$ for any prime $p$. We have

$$
D_{2}\left(k_{i}\right) \rightarrow 0
$$

if and only if $\lim _{i \rightarrow \infty} \sum_{p \mid k_{i}} 1 / p \rightarrow \infty$. Moreover,

$$
D_{2}\left(k_{i}\right) \rightarrow 1
$$

if and only if $\lim _{i \rightarrow \infty} \sum_{p \mid k_{i}} 1 / p \rightarrow 0$. The sums are taken over all primes $p$ which divide $k_{i}$.

In light of Remark 3, one may ask which values in the interval $\left[D_{n}(0), 1\right]$ can be obtained as partial limits of the function $D_{n}$. In this direction, we have the following result.

Proposition 5. For $n \geq 4$, the set of values of $D_{n}(k)$ as $k$ ranges over $\mathbb{Z}$ is not dense in in the interval $\left[D_{n}(0), 1\right]$. For $n=2$, the set of values of $D_{2}(k)$ as $k$ ranges over $\mathbb{Z}$ is dense in the interval $[0,1]$.

Section 4 is dedicated to the proofs of Theorem 2, Proposition 4 and Proposition 5. 


\subsection{Proof outline of Theorem 1}

Our proof of Theorem 1 uses essentially the same approach as [DRS93]. The set $M_{n, k}^{\prime}$ is partitioned into a finite number of orbits $A \mathrm{SL}_{n}(\mathbb{Z})$, where $A \in M_{n, k}$ are matrices in Hermite normal form with primitive row vectors. We count the matrices in each orbit separately. The number of matrices in each orbit scales as a fraction $1 / k^{n-1}$ of the number of matrices in $\mathrm{SL}_{n}(\mathbb{Z})$. We can view $\mathrm{SL}_{n}(\mathbb{Z})$ as a lattice in the space $\mathrm{SL}_{n}(\mathbb{R})$, and the problem is reduced to a lattice point counting problem. The lattice points inside the ball $B_{T}$ are counted by evaluating the normalized Haar measure of $B_{T} \cap \mathrm{SL}_{n}(\mathbb{R})$.

\section{Preliminaries}

The Riemann zeta function $\zeta$ is given by

$$
\zeta(s):=\sum_{n=1}^{\infty} \frac{1}{n^{s}}=\prod_{p} \frac{1}{1-1 / p^{s}}
$$

for $\operatorname{Re} s>1$, where we use the convention that when an index $p$ is used in a sum or product, it ranges over the set of primes.

The Möbius function $\mu$ is defined by $\mu(k):=(-1)^{m}$ if $k$ is a product of $m$ distinct prime factors (that is, $k$ is square-free), and $\mu(k):=0$ otherwise. We note that $\mu$ is a multiplicative function, that is, a function $f: \mathbb{N}^{*} \rightarrow \mathbb{C}$ defined on the positive integers such that $f(a b)=f(a) f(b)$ for all coprime $a, b$.

We will use the fact that $\mathrm{SL}_{n}(\mathbb{R})=M_{n, 1}$ has a normalized Haar measure $\nu$ which is bi-invariant (see [Sie45]).

\subsection{Lattice point counting}

Let $G$ be a topological group with a normalized Haar measure $\nu_{G}$ and a lattice $\Gamma \subseteq G$, and let $G_{T}$ be an increasing family of bounded subsets of $G$ for all $T \geq 1$. Under certain conditions (see for instance GN10), we have

$$
\left|G_{T} \cap \Gamma\right| \sim \nu_{G}\left(G_{T} \cap G\right),
$$

where we by $f(T) \sim g(T)$ mean that $f(T) / g(T) \rightarrow 1$ as $T \rightarrow \infty$. In this paper, we are interested in the lattice $\mathrm{SL}_{n}(\mathbb{Z})$ inside $\mathrm{SL}_{n}(\mathbb{R})$, and the following result will be crucial.

Theorem 6 [DRS93], Theorem 1.10). Let $B_{T}$ be the ball of radius $T$ in the space $M_{n}(\mathbb{R})$ of real $n \times n$-matrices under the Euclidean norm $\|A\|=\sqrt{\operatorname{tr}\left(A^{t} A\right)}$. Let $\nu$ be the normalized Haar measure of $\mathrm{SL}_{n}(\mathbb{R})$. Then

$$
\left|B_{T} \cap \mathrm{SL}_{n}(\mathbb{Z})\right|=\nu\left(B_{T} \cap \mathrm{SL}_{n}(\mathbb{R})\right)+O_{\varepsilon}\left(T^{n(n-1)-1 /(n+1)+\varepsilon}\right)
$$

for all $\varepsilon>0$, and the main term is given by

$$
\left|B_{T} \cap \mathrm{SL}_{n}(\mathbb{Z})\right| \sim C_{1} T^{n(n-1)}, \quad C_{1}=\frac{1}{\zeta(2) \cdots \zeta(n)} \frac{\pi^{n^{2} / 2}}{\Gamma\left(\frac{n}{2}\right) \Gamma\left(\frac{n(n-1)}{2}+1\right)} .
$$


In fact, a slightly more general statement is true. We can replace the balls $B_{T}$ in Theorem $\left[6\right.$ with balls under any norm on $M_{n}(\mathbb{R})$, and the asymptotics will still hold, save for a slighty worse exponent in the error term.

Theorem 7 [GN10], Corollary 2.3). Let $\|\cdot\|^{\prime}$ be any norm on the vector space $M_{n}(\mathbb{R})$, and let $G_{T}$ be the ball of radius $T$ in $M_{n}(\mathbb{R})$ under this norm. Let $\nu$ be the normalized Haar measure of $\mathrm{SL}_{n}(\mathbb{R})$. Then

$$
\left|G_{T} \cap \mathrm{SL}_{n}(\mathbb{Z})\right|=\nu\left(G_{T} \cap \mathrm{SL}_{n}(\mathbb{R})\right)+O_{\varepsilon}\left(T^{n(n-1)-1 /(2 n)+\varepsilon}\right)
$$

for all $\varepsilon>0$.

We will be interested in the following particular case of Theorem 7, Let $A \in M_{n, k}$. Then $\|X\|^{\prime}:=\left\|A^{-1} X\right\|$ defines a norm on $M_{n}(\mathbb{R})$, and the ball of radius $T$ in $M_{n}(\mathbb{R})$ under the norm $\|\cdot\|^{\prime}$ is $A \cdot B_{T}$.

Corollary 8. Let $A \in M_{n, k}$. Then

$$
\left|A B_{T} \cap \mathrm{SL}_{n}(\mathbb{Z})\right|=\nu\left(A B_{T} \cap \mathrm{SL}_{n}(\mathbb{R})\right)+O_{\varepsilon}\left(T^{n(n-1)-1 /(2 n)+\varepsilon}\right)
$$

for all $\varepsilon>0$, using the notation from Theorem 6 .

\section{The number of matrices with primitive rows}

In the present section, we will prove Theorem 1. We begin by noting that the common divisors of the entries of each row in an integer $n \times n$-matrix $A$ are preserved under multiplication on the right by any matrix $X \in \mathrm{SL}_{n}(\mathbb{Z})$. In particular, if each row of $A$ is primitive, then each row of $A X$ is primitive, for any $X \in \mathrm{SL}_{n}(\mathbb{Z})$. So we get:

Lemma 9. If $A \in M_{n, k}^{\prime}$ then $A X \in M_{n, k}^{\prime}$ for all $X \in \mathrm{SL}_{n}(\mathbb{Z})$. Thus $A \cdot \mathrm{SL}_{n}(\mathbb{Z}) \subseteq M_{n, k}^{\prime}$.

Consequently $M_{n, k}^{\prime}$ may be written as a disjoint union of orbits of $\mathrm{SL}_{n}(\mathbb{Z})$ :

$$
M_{n, k}^{\prime}=\bigcup_{A \in \mathcal{A}} A \mathrm{SL}_{n}(\mathbb{Z}),
$$

for properly chosen subsets $\mathcal{A}$ of $M_{n, k}^{\prime}$. In fact, as we will show in the following, the number of orbits is finite, and so we may take $\mathcal{A}$ to be finite.

A lower triangular integer matrix

$$
C:=\left(\begin{array}{cccc}
c_{11} & 0 & \cdots & 0 \\
c_{21} & c_{22} & \ddots & 0 \\
\vdots & & \ddots & 0 \\
c_{n 1} & \cdots & c_{n(n-1)} & c_{n n}
\end{array}\right)
$$

is said to be in (lower) Hermite normal form if $0<c_{11}$ and $0 \leq c_{i j}<c_{i i}$ for all $j<i$. The following result is well-known. 
Lemma 10 Coh93, Theorem 2.4.3). Assume $k>0$. Given an arbitrary matrix $A \in$ $M_{n, k}$, the orbit $A \mathrm{SL}_{n}(\mathbb{Z})$ contains a unique matrix $C$ in Hermite normal form.

We may thus write

$$
M_{n, k}^{\prime}=\bigcup_{i=1}^{m} A_{i} \mathrm{SL}_{n}(\mathbb{Z}),
$$

where $A_{1}, \ldots, A_{m}$ are the unique matrices in Hermite normal form with primitive row vectors and determinant $k$, and $m:=\left|M_{n, k}^{\prime} / \mathrm{SL}_{n}(\mathbb{Z})\right|$. By counting the number of matrices in Hermite normal form with determinant $k>0$, we get

$$
\left|M_{n, k} / \mathrm{SL}_{n}(\mathbb{Z})\right|=\sum_{d_{1} \cdots d_{n}=k} d_{1}^{0} d_{2}^{1} \cdots d_{n}^{n-1},
$$

where the sum ranges over all positive integer tuples $\left(d_{1}, \ldots, d_{n}\right)$ such that $d_{1} \cdots d_{n}=k$.

Proposition 11. Let $k>0$. Then

$$
\left|M_{n, k}^{\prime} / \mathrm{SL}_{n}(\mathbb{Z})\right|=\sum_{d_{1} \cdots d_{n}=k} \prod_{i=1}^{n} \sum_{g \mid d_{i}} \mu(g)\left(\frac{d_{i}}{g}\right)^{i-1}
$$

where the first sum ranges over all positive integer tuples $\left(d_{1}, \ldots, d_{n}\right)$ such that $d_{1} \cdots d_{n}=$ $k$.

Proof. We want to count those matrices in Hermite normal form which are in $M_{n, k}^{\prime}$, that is, $n \times n$-matrices in Hermite normal form with determinant $k$ and all rows primitive. The number of such matrices is

$$
\left|M_{n, k}^{\prime} / \mathrm{SL}_{n}(\mathbb{Z})\right|=\sum_{d_{1} \cdots d_{n}=k} \prod_{i=1}^{n} v_{i}\left(d_{i}\right),
$$

where $v_{i}(d)$ is the number of primitive vectors $\left(x_{1}, \ldots, x_{i-1}, d\right)$ such that $0 \leq x_{1}, \ldots, x_{i-1}<$ $d$. There is a bijective correspondence between the primitive vectors $\left(x_{1}, \ldots, x_{i-1}, d\right)$ and the vectors $y=\left(y_{1}, \ldots, y_{i-1}\right)$ such that $1 \leq y_{1}, \ldots, y_{i-1} \leq d$ and $\operatorname{gcd}(y)$ is coprime to $d$. Let $d=p_{1}^{a_{1}} \cdots p_{j}^{a_{j}}$ be the prime factorization of $d$. The number of vectors $y$ which are divisible by some set of primes $P \subseteq\left\{p_{1}, \ldots, p_{j}\right\}$ is

$$
\left(\frac{d}{\prod_{p \in P} p}\right)^{i-1}
$$

so by the principle of inclusion/exclusion (see [Sta97]), we have

$$
\begin{aligned}
& v_{i}(d)=\sum_{P \subseteq\left\{p_{1}, \ldots, p_{j}\right\}}(-1)^{|P|}\left(\frac{d}{\prod_{p \in P} p}\right)^{i-1} \\
= & \sum_{g \mid p_{1} \cdots p_{j}} \mu(g)\left(\frac{d}{g}\right)^{i-1}=\sum_{g \mid d} \mu(g)\left(\frac{d}{g}\right)^{i-1} .
\end{aligned}
$$


We are now ready to derive the asymptotics of $N_{n, k}^{\prime}(T)$.

Proof of Theorem 1, Let us write $A_{1}, \ldots, A_{m}$ for all the $n \times n$-matrices in Hermite normal form with determinant $k$, where $m:=\left|M_{n, k}^{\prime} / \mathrm{SL}_{n}(\mathbb{Z})\right|$, and let $1 \leq i \leq m$. Then

$$
\left|B_{T} \cap A_{i} \mathrm{SL}_{n}(\mathbb{Z})\right|=\left|A_{i}\left(A_{i}^{-1} B_{T} \cap \mathrm{SL}_{n}(\mathbb{Z})\right)\right|=\left|A_{i}^{-1} B_{T} \cap \mathrm{SL}_{n}(\mathbb{Z})\right|,
$$

which by Corollary 8 is equal to

$$
\nu\left(A_{i}^{-1} B_{T} \cap \mathrm{SL}_{n}(\mathbb{R})\right)+O_{\varepsilon}\left(T^{n(n-1)-1 /(2 n)+\varepsilon}\right)
$$

for any $\varepsilon>0$. Since $A_{i} / k^{1 / n} \in \mathrm{SL}_{n}(\mathbb{R})$, we get by the invariance of the measure $\nu$ that

$$
\begin{aligned}
& \nu\left(A_{i}^{-1} B_{T} \cap \mathrm{SL}_{n}(\mathbb{R})\right)=\nu\left(\frac{A_{i}}{k^{1 / n}}\left(A_{i}^{-1} B_{T} \cap \mathrm{SL}_{n}(\mathbb{R})\right)\right)= \\
& \nu\left(k^{-1 / n} B_{T} \cap \frac{A_{i}}{k^{1 / n}} \mathrm{SL}_{n}(\mathbb{R})\right)=\nu\left(B_{T / k^{1 / n}} \cap \mathrm{SL}_{n}(\mathbb{R})\right) .
\end{aligned}
$$

By Theorem 6, the last expression is equal to

$$
C_{1}\left(T / k^{1 / n}\right)^{n(n-1)}+O_{\varepsilon}\left(T^{n(n-1)-1 /(2 n)+\varepsilon}\right),
$$

and thus

$$
\left|B_{T} \cap A_{i} \mathrm{SL}_{n}(\mathbb{Z})\right|=\frac{C_{1}}{k^{n-1}} T^{n(n-1)}+O_{\varepsilon}\left(T^{n(n-1)-1 /(2 n)+\varepsilon}\right) .
$$

Now,

$$
N_{n, k}^{\prime}(T)=\left|B_{T} \cap M_{n, k}^{\prime}\right|=\left|B_{T} \cap \bigcup_{i=1}^{m} A_{i} \mathrm{SL}_{n}(\mathbb{Z})\right|=\sum_{i=1}^{m}\left|B_{T} \cap A_{i} \mathrm{SL}_{n}(\mathbb{Z})\right|,
$$

so applying (12) we get

$$
\begin{aligned}
& N_{n, k}^{\prime}(T)=\sum_{i=1}^{m} \frac{C_{1}}{k^{n-1}} T^{n(n-1)}+O_{\varepsilon}\left(T^{n(n-1)-1 /(2 n)+\varepsilon}\right)= \\
& \left|M_{n, k}^{\prime} / \mathrm{SL}_{n}(\mathbb{Z})\right| \frac{C_{1}}{k^{n-1}} T^{n(n-1)}+O_{\varepsilon}\left(T^{n(n-1)-1 /(2 n)+\varepsilon}\right),
\end{aligned}
$$

and we need only apply Proposition 11 to get an explicit constant for the main term. This concludes the proof. 


\section{Density of matrices with primitive rows}

Set

$$
\begin{aligned}
& a_{n}(k):=\left|M_{n, k} / \mathrm{SL}_{n}(\mathbb{Z})\right|=\sum_{d_{1} \cdots d_{n}=k} d_{1}^{0} \cdots d_{n}^{n-1}, \\
& a_{n}^{\prime}(k):=\left|M_{n, k}^{\prime} / \mathrm{SL}_{n}(\mathbb{Z})\right|=\sum_{d_{1} \cdots d_{n}=k} \prod_{i=1}^{n} \sum_{g \mid d_{i}} \mu(g)\left(\frac{d_{i}}{g}\right)^{i-1} .
\end{aligned}
$$

We would like to calculate the density of matrices with primitive rows in $M_{n, k}$ for $k \neq 0$, that is, the quantity

$$
D_{n}(k)=\lim _{T \rightarrow \infty} \frac{N_{n, k}^{\prime}(T)}{N_{n, k}(T)}=\frac{c_{n, k}^{\prime}}{c_{n, k}}=\frac{\left|M_{n, k}^{\prime} / \mathrm{SL}_{n}(\mathbb{Z})\right|}{\left|M_{n, k} / \mathrm{SL}_{n}(\mathbb{Z})\right|}=\frac{a_{n}^{\prime}(k)}{a_{n}(k)} .
$$

We will prove in section 4.1 that $a_{n}, a_{n}^{\prime}$ and $D_{n}$ are multiplicative functions, and therefore we need only understand their behavior for prime powers $k=p^{m}$. We will now prove a sequence of lemmas which we will finally use in section 4.2 to prove Theorem 2 .

Lemma 15. The functions $a_{n}^{\prime}$ and $a_{n}$ are connected via the identity

$$
a_{n}^{\prime}\left(p^{m}\right)=\sum_{i=0}^{m}(-1)^{i}\left(\begin{array}{c}
n \\
i
\end{array}\right) a_{n}\left(p^{m-i}\right)
$$

for primes $p$ and $m \geq 0$.

Proof. $a_{n}\left(p^{m}\right)$ counts the number of $n \times n$-matrices in Hermite normal form with determinant $p^{m}$, whereas $a_{n}^{\prime}\left(p^{m}\right)$ counts the number of such with primitive rows. If $A$ is a matrix in $M_{n, k} \backslash M_{n, k}^{\prime}$, then some set of rows, indexed by $S \subseteq[n]:=\{1, \ldots, n\}$ (where $|S| \leq m)$, are divisible by $p$. The number of such matrices is $a_{n}\left(p^{m-|S|}\right)$, and thus by the inclusion/exclusion principle,

$$
a_{n}^{\prime}\left(p^{m}\right)=\sum_{\substack{S \subseteq[n] \\
|S| \leq m}}(-1)^{|S|} a_{n}\left(p^{m-|S|}\right)=\sum_{i=0}^{m}(-1)^{i}\left(\begin{array}{c}
n \\
i
\end{array}\right) a_{n}\left(p^{m-i}\right)
$$

Lemma 16. For any prime $p$ and $m \geq 1$, the following recursion holds:

$$
a_{n}\left(p^{m}\right)=p^{n-1} a_{n}\left(p^{m-1}\right)+a_{n-1}\left(p^{m}\right),
$$

or equivalently,

$$
a_{n}\left(p^{m-1}\right)=\frac{a_{n}\left(p^{m}\right)-a_{n-1}\left(p^{m}\right)}{p^{n-1}} .
$$


Proof. We split the sum

$$
a_{n}\left(p^{m}\right)=\sum_{d_{1} \cdots d_{n}=p^{m}} d_{1}^{0} \cdots d_{n}^{n-1}
$$

into two parts, one part where $d_{n}$ is divisible by $p$, and another part where it is not (so that $d_{n}=1$ ). The terms corresponding to $d_{n}=1$ sum to $a_{n-1}\left(p^{m}\right)$. Where $d_{n}$ is divisible by $p$, we can write $d_{n}=: p e_{n}$ for some $e_{n}$. Let $e_{i}:=d_{i}$ for all $i<n$. Thus,

$$
\sum_{\substack{d_{1} \cdots d_{n}=p^{m} \\ p \mid d_{n}}} d_{1}^{0} \cdots d_{n}^{n-1}=\sum_{e_{1} \cdots e_{n}=p^{m-1}} e_{1}^{0} \cdots\left(p e_{n}\right)^{n-1}=p^{n-1} a_{n}\left(p^{m-1}\right) .
$$

Adding the two parts gives us $a_{n}\left(p^{m}\right)=p^{n-1} a_{n}\left(p^{m-1}\right)+a_{n-1}\left(p^{m}\right)$, from which the second claim in the lemma follows by rearrangement.

Lemma 17. Let $n$ and $p$ be fixed, where $n \geq 3$ and $p$ is a prime. Then

$$
D_{n}\left(p^{m}\right) \rightarrow\left(1-\frac{1}{p^{n-1}}\right)^{n}
$$

as $m \rightarrow \infty$.

Proof. We apply the simple upper bound

$$
a_{n-1}\left(p^{m}\right)=\sum_{d_{1} \cdots d_{n-1}=p^{m}} d_{1}^{0} \cdots d_{n}^{n-2} \leq \sum_{d_{1} \cdots d_{n-1}=p^{m}}\left(p^{m}\right)^{n-2}=(m+1)^{n-1}\left(p^{m}\right)^{n-2}
$$

to the expression for $a_{n}\left(p^{m-1}\right)$ in Lemma 16 .

$$
\begin{aligned}
a_{n}\left(p^{m-1}\right) & =\frac{1}{p^{n-1}}\left(a_{n}\left(p^{m}\right)-a_{n-1}\left(p^{m}\right)\right) \\
& =\frac{1}{p^{n-1}} a_{n}\left(p^{m}\right)+O\left(\left(p^{m}\right)^{n-2}(m+1)^{n-1}\right) .
\end{aligned}
$$

Repeated application (at most $n$ times) of this formula yields the asymptotics

$$
a_{n}\left(p^{m-i}\right)=\frac{1}{\left(p^{n-1}\right)^{i}} a_{n}\left(p^{m}\right)+O\left(\left(p^{m}\right)^{n-2}(m+1)^{n-1}\right)
$$

for $1 \leq i \leq n$.

Now let $m \rightarrow \infty$, so that we may assume $m$ to be larger than $n$. The sum in Lemma 15 then extends up to $i=n$ (because the factors $\left(\begin{array}{l}n \\ i\end{array}\right)$ vanish for larger $i$ ), so

$$
\begin{aligned}
a_{n}^{\prime}\left(p^{m}\right) & =\sum_{i=0}^{n}(-1)^{i}\left(\begin{array}{c}
n \\
i
\end{array}\right) a_{n}\left(p^{m-i}\right) \\
& =\sum_{i=0}^{n}(-1)^{i}\left(\begin{array}{c}
n \\
i
\end{array}\right) \frac{1}{\left(p^{n-1}\right)^{i}} a_{n}\left(p^{m}\right)+O\left(\left(p^{m}\right)^{n-2}(m+1)^{n-1}\right) .
\end{aligned}
$$


We divide by $a_{n}\left(p^{m}\right)$ on both sides and use the fact that $a_{n}\left(p^{m}\right) \geq\left(p^{m}\right)^{n-1}$, so that

$$
\begin{aligned}
D_{n}\left(p^{m}\right) & =\sum_{i=0}^{n}(-1)^{i}\left(\begin{array}{c}
n \\
i
\end{array}\right) \frac{1}{\left(p^{n-1}\right)^{i}}+O\left(\frac{\left(p^{m}\right)^{n-2}(m+1)^{n-1}}{\left(p^{m}\right)^{n-1}}\right) \\
& =\sum_{i=0}^{n}\left(\begin{array}{c}
n \\
i
\end{array}\right)\left(\frac{-1}{p^{n-1}}\right)^{i}+O\left(\frac{(m+1)^{n-1}}{p^{m}}\right) \\
& =\left(1-\frac{1}{p^{n-1}}\right)^{n}+O\left(\frac{(m+1)^{n-1}}{p^{m}}\right) .
\end{aligned}
$$

As $m \rightarrow \infty$, the second term on the right vanishes.

\subsection{Multiplicativity and monotonicity of the density function}

In this section we will prove the following proposition.

Proposition 18. The function $D_{n}$ is multiplicative, and $D_{n}\left(p^{m}\right)$ is strictly decreasing as a function of $m$ for any fixed prime $p$ and dimension $n \geq 2$.

We may rewrite (13) as

$$
a_{n}=(\cdot)^{n-1} * \cdots *(\cdot)^{0}
$$

where $(\cdot)^{i}$ is the function $x \mapsto x^{i}$ and $*$ denotes the Dirichlet convolution. Similarly, we may rewrite (14) as

$$
a_{n}^{\prime}=\left(\mu *(\cdot)^{n-1}\right) * \cdots *\left(\mu *(\cdot)^{0}\right),
$$

so by the commutativity and associativity of the Dirichlet convolution we have

$$
a_{n}^{\prime}=\mu^{* n} * a_{n}
$$

where $\mu^{* n}$ denotes the convolution of $\mu$ with itself $n$ times (so that $\mu^{* 1}=\mu$ ). Since the Dirichlet inverse of $\mu$ is the constant function 1, we have also the relation

$$
a_{n}=1^{* n} * a_{n}^{\prime} .
$$

As $\mu$ and $(\cdot)^{i}$ are multiplicative functions, it follows that $a_{n}, a_{n}^{\prime}$ and $D_{n}$ are multiplicative as well.

Now, we want to show that $D_{n}\left(p^{m}\right)=a_{n}^{\prime}\left(p^{m}\right) / a_{n}\left(p^{m}\right)$ is strictly decreasing as a function of $m$, for fixed $n \geq 2$ and primes $p$, or equivalently that

$$
\frac{a_{n}^{\prime}\left(p^{m}\right)}{a_{n}\left(p^{m}\right)}>\frac{a_{n}^{\prime}\left(p^{m+1}\right)}{a_{n}\left(p^{m+1}\right)}
$$

for all $m \geq 0$. The inequality (201) is equivalent to

$$
\frac{a_{n}^{\prime}\left(p^{m}\right)}{\left(1^{* n} * a_{n}^{\prime}\right)\left(p^{m}\right)}>\frac{a_{n}^{\prime}\left(p^{m+1}\right)}{\left(1^{* n} * a_{n}^{\prime}\right)\left(p^{m+1}\right)}
$$


for all $m \geq 0$, which is equivalent to

$$
\frac{a_{n}^{\prime}\left(p^{m}\right)}{\sum_{i=0}^{m} 1^{* n}\left(p^{i}\right) a_{n}^{\prime}\left(p^{m-i}\right)}>\frac{a_{n}^{\prime}\left(p^{m+1}\right)}{\sum_{i=0}^{m+1} 1^{* n}\left(p^{i}\right) a_{n}^{\prime}\left(p^{m+1-i}\right)},
$$

or, after taking the reciprocal of both sides,

$$
\sum_{i=0}^{m} 1^{* n}\left(p^{i}\right) \frac{a_{n}^{\prime}\left(p^{m-i}\right)}{a_{n}^{\prime}\left(p^{m}\right)}<\sum_{i=0}^{m+1} 1^{* n}\left(p^{i}\right) \frac{a_{n}^{\prime}\left(p^{m+1-i}\right)}{a_{n}^{\prime}\left(p^{m+1}\right)} .
$$

Since the last term $(i=m+1)$ on the right hand side is positive, this inequality holds if

$$
\frac{a_{n}^{\prime}\left(p^{m-i}\right)}{a_{n}^{\prime}\left(p^{m}\right)} \leq \frac{a_{n}^{\prime}\left(p^{m+1-i}\right)}{a_{n}^{\prime}\left(p^{m+1}\right)}
$$

for all $i \leq m$. We can rearrange this inequality as

$$
\frac{a_{n}^{\prime}\left(p^{m+1}\right)}{a_{n}^{\prime}\left(p^{m}\right)} \leq \frac{a_{n}^{\prime}\left(p^{m+1-i}\right)}{a_{n}^{\prime}\left(p^{m-i}\right)},
$$

which states that $a_{n}^{\prime}\left(p^{m+1}\right) / a_{n}^{\prime}\left(p^{m}\right)$ is a non-increasing function of $m$, for fixed $n \geq 2$ and $p$ prime. We will therefore be done if we can prove that

$$
a_{n}^{\prime}\left(p^{m+1}\right) a_{n}^{\prime}\left(p^{m+1}\right) \geq a_{n}^{\prime}\left(p^{m}\right) a_{n}^{\prime}\left(p^{m+2}\right)
$$

for all $m \geq 0$, or equivalently, that the function $m \mapsto a_{n}^{\prime}\left(p^{m}\right)$ is logarithmically concave:

We say that a sequence $u: \mathbb{N}_{0} \rightarrow \mathbb{R}$ is logarithmically concave if

$$
u_{r}^{2}-u_{r-1} u_{r+1} \geq 0
$$

for all $r \geq 1$. We note that a sequence $u$ of positive real numbers is logarithmically concave if and only if $u_{1} / u_{0} \geq u_{2} / u_{1} \geq u_{3} / u_{2} \geq \cdots$, that is, if and only if $\left(u_{1} / u_{0}, u_{2} / u_{1}, u_{3} / u_{2}, \ldots\right)$ is a non-increasing sequence. Also note that if $u$ is positive and logarithmically concave, then the inequality $u_{i+1} / u_{i} \geq u_{j+1} / u_{j}$ implies the inequality $u_{i+1} u_{j}-u_{j+1} u_{i} \geq 0$ for all indices $i<j$.

Let $\star$ denote the discrete convolution, so that $(u \star v)_{r}=\sum_{j=0}^{r} u_{r-j} v_{j}$ for all $r \geq 0$ given any sequences $u, v: \mathbb{N}_{0} \rightarrow \mathbb{R}$. We will need the following fact, which follows from the proof of Theorem 1 in [Men69].

Theorem 22 [Men69, Theorem 1). Let $u, v: \mathbb{N}_{0} \rightarrow \mathbb{R}$ be sequences such that $u_{0}=v_{0}=$ 1 , and let $w=u \star v$. Then we may write $w_{r}^{2}-w_{r-1} w_{r+1}=\mathrm{I}+\mathrm{II}+\mathrm{III}$, where

$$
\begin{aligned}
\mathrm{I} & =\sum_{0 \leq i<j \leq r-1}\left(v_{j} v_{i+1}-v_{j+1} v_{i}\right)\left(u_{r-j} u_{r-i-1}-u_{r-1-j} u_{r-i}\right), \\
\mathrm{II} & =\sum_{j=0}^{r-1} v_{j}\left(u_{r-j} u_{r}-u_{r-1-j} u_{r+1}\right), \\
\mathrm{III} & =v_{r} u_{r}+\sum_{j=0}^{r-1} u_{j}\left(v_{r} v_{r-j}-v_{r+1} v_{r-1-j}\right),
\end{aligned}
$$


for all $r \geq 1$. In particular, if $u, v$ are positive and logarithmically concave sequences, then so is $w$, since all factors in the sums in I, II, III are non-negative for such $u, v$.

Fix $n$ and $p$. Then since $\left(\mu *(\cdot)^{i}\right)\left(p^{m}\right)=\sum_{r=0}^{m} \mu\left(p^{m-r}\right) p^{r i}=\left(M \star P_{i}\right)(m)$ where $M$ is the sequence $(1,-1,0,0,0, \ldots)$ and where $P_{i}$ is the sequence $\left(1, p^{i}, p^{2 i}, p^{3 i}, \ldots\right)$, equation (19) implies that the function $m \mapsto a_{n}^{\prime}\left(p^{m}\right)$ can be written as

$$
\left(M \star P_{n-1}\right) \star \cdots \star\left(M \star P_{0}\right) .
$$

Lemma 23. Let $0 \leq i<j$. Then $\left(M \star P_{i}\right) \star\left(M \star P_{j}\right)$ is positive and logarithmically concave if and only if $i>0$.

Proof. Write $u:=M \star P_{i}$ and $v:=M \star P_{j}$ where $i<j$. We have $u_{0}=1$ and $u_{r}=$ $p^{i r}-p^{i(r-1)}$ for all $r \geq 1$. Thus $u_{1} u_{r}-u_{0} u_{r+1}=\left(p^{i}-1\right)\left(p^{i r}-p^{i(r-1)}\right)-\left(p^{i(r+1)}-p^{i r}\right)=$ $p^{i(r-1)}-p^{i r}=-u_{r}$ for all $r \geq 1$, and $u_{s} u_{r}-u_{s-1} u_{r+1}=0$ when $s \geq 2, r \geq 1$ or $s=1, r=0$. Likewise $v_{s} v_{r}-v_{s-1} v_{r+1}$ is $-v_{r}$ if $s=1, r \geq 1$, and 0 otherwise.

Let $w:=u \star v=\left(M \star P_{i}\right) \star\left(M \star P_{j}\right)$. By Theorem 22 we can write $w_{r}^{2}-w_{r-1} w_{r+1}=$ $\mathrm{I}+\mathrm{II}+\mathrm{III}$, where

$$
\begin{aligned}
\mathrm{I} & =\left(-v_{r-1}\right)\left(-u_{r-1}\right), \\
\mathrm{II} & =v_{r-1}\left(-u_{r}\right), \\
\mathrm{III} & =v_{r} u_{r}+u_{r-1}\left(-v_{r}\right),
\end{aligned}
$$

for all $r \geq 1$, and therefore

$$
\begin{gathered}
w_{r}^{2}-w_{r-1} w_{r+1}=u_{r-1} v_{r-1}+u_{r} v_{r}-u_{r} v_{r-1}-u_{r-1} v_{r}= \\
\left(u_{r}-u_{r-1}\right)\left(v_{r}-v_{r-1}\right) .
\end{gathered}
$$

Thus, since $\left(u_{0}, u_{1}, \ldots\right)$ is a non-decreasing sequence for $i>0$, and likewise $\left(v_{0}, v_{1}, \ldots\right)$ is a non-decreasing sequence for $j>0$, we get $w_{r}^{2}-w_{r-1} w_{r+1} \geq 0$ for all $r \geq 1$ for $i>0$. Also, the sequence $w$ is positive for $i, j>0$ since it is then the convolution of two positive sequences. If $i=0$, then the inequality $w_{r}^{2}-w_{r-1} w_{r+1} \geq 0$ fails for $r=1$ since then $u_{1}-u_{0}=\left(p^{0}-1\right)-1<0$ and $v_{1}-v_{0}=\left(p^{j}-1\right)-1>0$.

We will prove Proposition 18 by induction on $n$. The base case is the following proposition, which we will prove in Appendix B.

Proposition 24. For $n=4,5$ and any fixed prime $p$, the function $m \mapsto a_{n}^{\prime}\left(p^{m}\right)$ is logarithmically concave.

It happens that $a_{n}^{\prime}\left(p^{m}\right)$, as a function of $m$, is not logarithmically concave for $n=2$ or $n=3$ for all $p$ (it fails the inequality (21) for $r=1$ when $p=2$ ), so we will also need the following proposition, which we prove in Appendix $\mathrm{A}$.

Proposition 25. For $n=2,3$ and any fixed prime $p$, the function $m \mapsto D_{n}\left(p^{m}\right)$ is strictly decreasing. 
The proofs of Propositions 25 and 24 consist of explicitly evaluating $a_{n}\left(p^{m}\right)$ and $a_{n}^{\prime}\left(p^{m}\right)$ for the values of $n$ in question, both of which are polynomials in $p$ with exponents in $m$, and verifying equations (20) and (21), respectively.

Proof of Proposition [18. By Proposition 24] and Proposition 25, it suffices to consider $n>5$. By Proposition 24 and Theorem 22, it follows that $A_{n}^{\prime}(m):=a_{n}^{\prime}\left(p^{m}\right)$ is logarithmically concave for all $n>5$ and any $p$, since for any even $n>5$, we can write

$$
A_{n}^{\prime}=A_{4}^{\prime} \star\left[\left(M \star P_{4}\right) \star\left(M \star P_{5}\right)\right] \star \cdots \star\left[\left(M \star P_{n-2}\right) \star\left(M \star P_{n-1}\right)\right],
$$

and for any odd $n>5$, we can write

$$
A_{n}^{\prime}=A_{5}^{\prime} \star\left[\left(M \star P_{5}\right) \star\left(M \star P_{6}\right)\right] \star \cdots \star\left[\left(M \star P_{n-2}\right) \star\left(M \star P_{n-1}\right)\right],
$$

and in both cases we have written $A_{n}^{\prime}$ as the convolution of positive and logarithmically concave sequences, by Lemma 23. We have thus proven the inequality (21), and this concludes the proof of Proposition 18,

\subsection{Asymptotics of the density function}

In this section we prove Theorem 2 and thus derive the asymptotics of $D_{n}(k)$. Fix $n \geq 3$. For any nonzero integer $k_{i}$, write $k_{i}=\prod_{p} p^{m_{p}(i)}$ as a product of prime powers, where all but finitely many of the exponents $m_{p}(i)$ are zero. Then since $D_{n}$ is multiplicative, we have

$$
D_{n}\left(k_{i}\right)=\prod_{p} D_{n}\left(p^{m_{p}(i)}\right) .
$$

Now, by Lemma 17 and Proposition 18, we get

$$
1 \geq \prod_{p} D_{n}\left(p^{m_{p}(i)}\right)>\prod_{p}\left(1-\frac{1}{p^{n-1}}\right)^{n}=\frac{1}{\zeta(n-1)^{n}}>0,
$$

so it follows by dominated convergence that

$$
\lim _{i \rightarrow \infty} \prod_{p} D_{n}\left(p^{m_{p}(i)}\right)=\prod_{p} \lim _{i \rightarrow \infty} D_{n}\left(p^{m_{p}(i)}\right) .
$$

whenever $\left(k_{1}, k_{2}, \ldots\right)$ is a sequence of nonzero integers such that the $\operatorname{limit}_{\lim _{i \rightarrow \infty}} D_{n}\left(p^{m_{p}(i)}\right)$ exists for each prime $p$.

Let $\left(k_{1}, k_{2}, \ldots\right)$ be a sequence of nonzero integers. It now follows from (26), Proposition 18 and the fact that $D_{n}(1)=1$, that

$$
D_{n}\left(k_{i}\right) \rightarrow 1
$$

if and only if $m_{p}(i) \rightarrow 0$ as $i \rightarrow \infty$ for all $p$, that is, if and only if $\left(k_{1}, k_{2}, \ldots\right)$ is a rough sequence. Likewise it follows, using Lemma 17, that

$$
D_{n}\left(k_{i}\right) \rightarrow \frac{1}{\zeta(n-1)^{n}}
$$


if and only if $m_{p}(i) \rightarrow \infty$ for all $p$, that is, if and only if $\left(k_{1}, k_{2}, \ldots\right)$ is a totally divisible sequence. Since $D_{n}(0)=1 / \zeta(n-1)^{n}$, we may allow the elements of the sequence $\left(k_{1}, k_{2}, \ldots\right)$ to also assume the value 0 .

Finally, it follows that $D_{n}(k) \rightarrow 1$ as $n \rightarrow \infty$ uniformly with respect to $k$ since

$$
D_{n}(k) \geq \frac{1}{\zeta(n-1)^{n}} \rightarrow 1
$$

as $n \rightarrow \infty$ because $\zeta(n-1)=1+O\left(2^{-n}\right)$ for $n \geq 3$. We have thus proved all parts of Theorem 2 ,

We conclude this section by proving Proposition 4, which tells us the asymptotics of $D_{2}(k)$ for $n=2$.

Proof of Proposition 布. If $m=0$, we have $D_{2}\left(p^{m}\right)=1$. Assume $m>0$. The $2 \times 2$ matrices in Hermite normal form with determinant $p^{m}$ and primitive rows are of the form $\left(\begin{array}{cc}1 & 0 \\ x & p^{m}\end{array}\right)$ where $0 \leq x<p^{m}, p \nmid x$. Thus $a_{2}^{\prime}\left(p^{m}\right)=p^{m}(1-1 / p)$. Moreover,

$$
a_{2}\left(p^{m}\right)=\sum_{d_{1}} d_{2}=p^{m}=\sum_{i+j=m} p^{i}=\sum_{i=0}^{m} p^{i}=\frac{p^{m+1}-1}{p-1}=p^{m} \frac{1-1 / p^{m+1}}{1-1 / p}
$$

so

$$
D_{2}\left(p^{m}\right)=\frac{(1-1 / p)^{2}}{1-1 / p^{m+1}}
$$

for all $m \geq 1$. We see immediately that $D_{2}\left(p^{m}\right)$ is strictly decreasing as a function of $m$, for any fixed $p$. Therefore

$$
\left(1-\frac{1}{p}\right)^{2} \leq D_{2}\left(p^{m}\right) \leq 1-\frac{1}{p}
$$

Since $D_{2}$ is multiplicative, we get

$$
\left[\prod_{p \mid k}\left(1-\frac{1}{p}\right)\right]^{2} \leq D_{2}(k) \leq \prod_{p \mid k}\left(1-\frac{1}{p}\right) .
$$

The left and right sides both tend to 0 if and only if $\lim _{i \rightarrow \infty} \sum_{p \mid k_{i}} 1 / p \rightarrow \infty$, and they both converge to 1 if and only if $\lim _{i \rightarrow \infty} \sum_{p \mid k_{i}} 1 / p \rightarrow 0$.

\subsection{The image of the density function}

Proof of Proposition 5 for $n \geq 4$. By Proposition 18, the function $D_{n}$ is multiplicative, and $D_{n}\left(p^{m}\right)$ is strictly decreasing as a function of $m$ for any fixed $p, n$. Thus we get 
$D_{n}(k) \leq D_{n}(2)$ whenever $k$ is divisible by 2 . When $k$ is not divisible by 2 , we get

$$
\begin{gathered}
D_{n}(k) \geq \prod_{p \geq 3} \lim _{m \rightarrow \infty} D_{n}\left(p^{m}\right)=\prod_{p \geq 3}\left(1-1 / p^{n-1}\right)^{n}= \\
\frac{1}{\left(1-1 / 2^{n-1}\right)^{n}} \prod_{p}\left(1-1 / p^{n-1}\right)^{n}=\frac{1}{\left(1-1 / 2^{n-1}\right)^{n}} \frac{1}{\zeta(n-1)^{n}} .
\end{gathered}
$$

by Lemma 17. We will show that this value is larger than $D_{n}(2)$, which will prove that the image of $D_{n}: \mathbb{Z} \rightarrow \mathbb{R}$ is not dense in $\left[D_{n}(0), 1\right]$. By equation (13) we have $a_{n}(2)=$ $\sum_{i=1}^{n} 2^{i-1}=2^{n}-1$ and by Lemma 15 we have $a_{n}^{\prime}(2)=a_{n}(2)-n a_{n}(1)=\left(2^{n}-1\right)-n$, so $D_{n}(2)=1-n /\left(2^{n}-1\right)$.

Thus it suffices to prove

$$
\frac{1}{\left(1-1 / 2^{n-1}\right)^{n}} \frac{1}{\zeta(n-1)^{n}}>1-\frac{n}{2^{n}-1} .
$$

This inequality can be verified numerically for $n=4,5$. Let us now assume $n \geq 6$. The inequality (28) is is equivalent to

$$
-\log \left(1-n /\left(2^{n}-1\right)\right)-n \log \left(1-1 / 2^{n-1}\right)>n \log \zeta(n-1) .
$$

By Taylor expansion, the first term on the left hand side is $>n /\left(2^{n}-1\right)>n / 2^{n}$, and the second term on the left hand side is $>n / 2^{n-1}$. Thus the inequality above follows from $1 / 2^{n}+1 / 2^{n-1} \geq \log \zeta(n-1)$, or equivalently $e^{3 / 2^{n}} \geq \zeta(n-1)$. We bound the left hand side from below by $1+3 / 2^{n}$, and we bound the right hand side from above by $1+1 / 2^{n-1}+\int_{2}^{\infty} \frac{d x}{x^{n-1}}$. Thus the inequality follows from $1+3 / 2^{n} \geq 1+1 / 2^{n-1}+1 /((n-$ 2) $2^{n-2}$ ) or equivalently $3 \geq 2+4 /(n-2)$, which is true for all $n \geq 6$.

Proof of Proposition 5 for $n=2$. It suffices to show that the set of values of $-\log \left(D_{2}(k)\right)$ as $k$ ranges over positive square-free integers is dense in $[0, \infty)$. By the identity (27) we have

$$
D_{2}(p)=\frac{(1-1 / p)^{2}}{1-1 / p^{2}}=\frac{1-1 / p}{1+1 / p}=\frac{p-1}{p+1}=1-\frac{2}{p+1} .
$$

Let $k>0$ be squarefree, and let $P_{0}$ be the set of primes dividing $k$. Then

$$
-\log D_{2}(k)=-\log \prod_{p \in P_{0}} D_{2}(p)=\sum_{p \in P_{0}}\left(-\log \left(1-\frac{2}{p+1}\right)\right) .
$$

The terms $d_{p}:=-\log \left(1-\frac{2}{p+1}\right)$ are positive, decreasing, and tend to zero as $p \rightarrow \infty$. By Taylor expansion, the sum $\sum_{p} d_{p}$ over all primes is larger than $\sum_{p} \frac{2}{p+1}$, which diverges since $\sum_{p} 1 / p$ diverges.

Now, given any $x \in[0, \infty)$ and any $\varepsilon>0$, we can find a $k$ such that $-\log D_{2}(k)$ is within a distance $\varepsilon$ from $x$ as follows. Let $p_{0}$ be the smallest prime such that $d_{p_{0}}<\varepsilon$, and let $P_{0}$ be the smallest set of consecutive primes, starting with $p_{0}$, such that $\sum_{p \in P_{0}} d_{p} \geq x$. Then the sum $\sum_{p \in P_{0}} d_{p}=-\log D_{2}(k)$ is at a distance at most $d_{p_{0}}<\varepsilon$ from $x$ since $d_{p}$ is decreasing, where $k=\prod_{p \in P_{0}} p$, and we are done. 


\section{A. Proof of Proposition 25}

We prove Proposition 25, Recall from equation (27) that $D_{2}\left(p^{m}\right)=(1-1 / p)^{2} /(1-$ $\left.1 / p^{m+1}\right)$ if $m>0$, and otherwise $D_{2}\left(p^{0}\right)=1$. Thus we see immediately that $D_{2}\left(p^{m}\right)$ is strictly decreasing as a function of $m$.

The case $n=3$ remains. By equation (13) we get

$$
\begin{gathered}
a_{3}\left(p^{m}\right)=\sum_{j_{1}+j_{2}+j_{3}=m} p^{j_{2}+2 j_{3}}=\sum_{j_{3}=0}^{m} p^{2 j_{3}} \sum_{j_{2}=0}^{m-j_{3}} p^{j_{3}}=\sum_{j_{3}=0}^{m} p^{2 j_{3}} \frac{1-p^{m-j_{3}+1}}{1-p}= \\
\frac{1}{1-p} \sum_{j_{3}=0}^{m}\left(p^{2 j_{3}}-p^{m+j_{3}+1}\right)=\frac{1}{1-p}\left(\frac{1-p^{2(m+1)}}{1-p^{2}}-p^{m+1} \frac{1-p^{m+1}}{1-p}\right)= \\
\frac{1-p^{2(m+1)}-(1+p) p^{m+1}+(1+p) p^{2(m+1)}}{(1-p)\left(1-p^{2}\right)}= \\
\frac{1-p^{m+1}-p^{m+2}+p^{(m+1)+(m+2)}}{(1-p)\left(1-p^{2}\right)}=\frac{\left(p^{m+1}-1\right)\left(p^{m+2}-1\right)}{(p-1)\left(p^{2}-1\right)} .
\end{gathered}
$$

for all $m \geq 1$. Let us write $I(P):=1$ if the condition $P$ is true, and $I(P):=0$ if the condition $P$ is false. By equation (14) we get for all $m \geq 1$ that

$$
\begin{gathered}
a_{3}^{\prime}\left(p^{m}\right)=\sum_{\substack{j_{1}, j_{2}, j_{3} \geq 0: \\
p^{j_{1}} p^{j_{2}} p^{j_{3}}=p^{m}}} \prod_{i=1}^{3} \sum_{\substack{r \geq 0: \\
p^{r} \mid p^{j_{i}}}} \mu\left(p^{r}\right)\left(p^{j_{i}-r}\right)^{i-1}= \\
\sum_{\substack{j_{2}, j_{3} \geq 0: \\
j_{2}+j_{3}=m}}\left(p^{j_{2}}-p^{j_{2}-1} I\left(j_{2}>0\right)\right)\left(p^{2 j_{3}}-p^{2\left(j_{3}-1\right)} I\left(j_{3}>0\right)\right) .
\end{gathered}
$$

We expand the product in the summand and split the sum into several geometric series which we sum individually. We get

$$
\begin{gathered}
\sum_{j_{2}=0}^{m}\left(p^{2 m-j_{2}}-p^{2 m-j_{2}-1} I\left(j_{2}>0\right)-p^{2 m-j_{2}-2} I\left(m>j_{2}\right)+p^{2 m-j_{2}-3} I\left(0<j_{2}<m\right)\right)= \\
p^{2 m}\left(\frac{1-p^{-(m+1)}}{1-p^{-1}}-p^{-1}\left(\frac{1-p^{-(m+1)}}{1-p^{-1}}-1\right)-p^{-2} \frac{1-p^{-m}}{1-p^{-1}}+p^{-3}\left(\frac{1-p^{-m}}{1-p^{-1}}-1\right)\right)= \\
\frac{p^{2 m}}{1-p^{-1}}\left(1-p^{-(m+1)}-p^{-1}\left(1-p^{-(m+1)}-\left(1-p^{-1}\right)\right)-\right. \\
\left.p^{-2}\left(1-p^{-m}\right)+p^{-3}\left(1-p^{-m}-\left(1-p^{-1}\right)\right)\right)= \\
\frac{p^{2 m}}{1-p^{-1}}\left(\left(1-p^{-2}\right)^{2}-p^{-m-1}\left(1-p^{-1}\right)^{2}\right)=\frac{p^{2 m}\left(1-p^{-2}\right)^{2}-p^{m-1}\left(1-p^{-1}\right)^{2}}{1-p^{-1}}= \\
\left(p^{2 m}(p+1)^{2}-p^{m+1}\right) \frac{p-1}{p^{3}} .
\end{gathered}
$$


Since $D_{3}(1)=1$ and $D_{3}\left(p^{m}\right)<1$ for all $m>0$ (the diagonal matrix with diagonal entries $1,1, p^{m}$ is in Hermite normal form, but its last row is not primitive), it suffices to show that $D_{3}\left(p^{m}\right)>D_{3}\left(p^{m+1}\right)$ for all $m \geq 1$. To see this, we note that

$$
\begin{gathered}
\frac{p^{2 m}(p+1)^{2}-p^{m+1}}{\left(p^{m+1}-1\right)\left(p^{m+2}-1\right)}>\frac{p^{2 m+2}(p+1)^{2}-p^{m+2}}{\left(p^{m+2}-1\right)\left(p^{m+3}-1\right)} \Longleftrightarrow \\
p^{2 m}(p+1)^{2}\left[\left(p^{m+3}-1\right)-p^{2}\left(p^{m+1}-1\right)\right]-p^{m+1}\left[\left(p^{m+3}-1\right)-p\left(p^{m+1}-1\right)\right]>0 \Longleftrightarrow \\
p^{2 m}(p+1)^{2}\left(p^{2}-1\right)-p^{m+1}\left(p^{m+2}+1\right)(p-1)>0 \Longleftrightarrow \\
p^{2 m}(p+1)^{3}-p^{2 m}\left(p^{3}+p^{1-m}\right)>0,
\end{gathered}
$$

where the last inequality is true since $(p+1)^{3}>p^{3}+1 \geq p^{3}+p^{1-m}$ for all $m \geq 1$ and all $p \geq 2$. This concludes the proof of Proposition 25 .

\section{B. Proof of Proposition 24}

\section{B.1. The case $n=4$}

We prove Proposition 24 for $n=4$. By equation (14), we can write

$$
\begin{aligned}
& a_{4}^{\prime}\left(p^{m}\right)=\sum_{\substack{j_{1}, j_{2}, j_{3}, j_{4} \geq 0: \\
p^{j_{1}} p^{j_{2}} p^{j_{3}} p^{j_{4}}=p^{m}}} \prod_{\substack{i=1 \\
p^{r} \mid p^{j_{i}}}}^{4} \sum_{\substack{r \geq 0 \\
p^{r}}} \mu\left(p^{r}\right)\left(p^{j_{i}-r}\right)^{i-1}= \\
& \sum_{j_{2}+j_{3}+j_{4}=m}\left(p^{j_{2}}-p^{j_{2}-1} I\left(j_{2}>0\right)\right)\left(p^{2 j_{3}}-p^{2\left(j_{3}-1\right)} I\left(j_{3}>0\right)\right)\left(p^{3 j_{4}}-p^{3\left(j_{4}-1\right)} I\left(j_{4}>0\right)\right),
\end{aligned}
$$

where $I(P)$ is defined as in (29). We evaluate this sum in the same way that we evaluated $a_{3}^{\prime}\left(p^{m}\right)$ in Appendix A: We expand the product in the summand and eliminate the symbols $I(P)$ by splitting the sum into several geometric series over different ranges, corresponding to the conditions $j_{2}>0$, and so on, and compute each geometric series individually. We assume $m \geq 1$ to guarantee that $\sum_{j_{2}=1}^{m}$, for instance, is never an empty sum. Thus, by a tedious but straightforward calculation, we get

$$
\begin{gathered}
a_{4}^{\prime}\left(p^{m}\right)=\frac{(p-1) p^{m-6}}{p+1}\left(p^{2 m}-p^{m+1}-4 p^{m+2}-6 p^{m+3}-4 p^{m+4}-p^{m+5}+3 p^{2 m+1}+\right. \\
\left.6 p^{2 m+2}+7 p^{2 m+3}+6 p^{2 m+4}+3 p^{2 m+5}+p^{2 m+6}+p^{3}\right) .
\end{gathered}
$$

Using this, one may show that

$$
\begin{gathered}
a_{4}^{\prime}\left(p^{m+1}\right)^{2}-a_{4}^{\prime}\left(p^{m}\right) a_{4}^{\prime}\left(p^{m+2}\right)= \\
(p-1)^{4} p^{3 m-7}\left((p+1)^{2}\left(p^{2}+p+1\right)^{3} p^{2 m}-\left(p^{2}+p+1\right)^{3} p^{m}+(p+1)^{2} p\right)= \\
(p-1)^{4} p^{3 m-7}\left(\left((p+1)^{2} p^{m}-1\right)\left(p^{2}+p+1\right)^{3} p^{m}+(p+1)^{2} p\right)
\end{gathered}
$$


which we see is positive for all $p \geq 2$ and all $m \geq 1$. Moreover, using $a_{4}^{\prime}\left(p^{0}\right)=1$, we get

$$
a_{4}^{\prime}\left(p^{1}\right)^{2}-a_{4}^{\prime}\left(p^{0}\right) a_{4}^{\prime}\left(p^{2}\right)=(p-1)(p+2)\left(p^{3}-3\right),
$$

which is positive for all $p \geq 2$. Thus we have proved the inequality (21) for all $m \geq 0$, which completes the proof of Proposition 24 for the case $n=4$.

Equations (30) and (31) may be verified with a computer algebra system, for instance with the Mathematica code provided at

$$
\text { http://www.math.kth.se/ ^holmin/files/x/a4prime_is_logconcave. }
$$

\section{B.2. The case $n=5$}

We prove Proposition 24 for $n=5$. We repeat the procedure above. We evaluate

$$
\begin{aligned}
a_{5}^{\prime}\left(p^{m}\right)= & \sum_{j_{2}+j_{3}+j_{4}+j_{5}=m}\left(p^{j_{2}}-p^{j_{2}-1} I\left(j_{2}>0\right)\right)\left(p^{2 j_{3}}-p^{2\left(j_{3}-1\right)} I\left(j_{3}>0\right)\right) \times \\
& \times\left(p^{3 j_{4}}-p^{3\left(j_{4}-1\right)} I\left(j_{4}>0\right)\right)\left(p^{4 j_{5}}-p^{4\left(j_{5}-1\right)} I\left(j_{4}>0\right)\right) .
\end{aligned}
$$

As before, we expand the product in the summand, and split the sum into several geometric series. This yields $a_{5}^{\prime}\left(p^{m}\right)=\frac{p-1}{p^{10}(p+1)\left(p^{2}+p+1\right)}\left(p^{4 m}-p^{m+6}+p^{2 m+3}+5 p^{2 m+4}+\right.$ $11 p^{2 m+5}+14 p^{2 m+6}+11 p^{2 m+7}+5 p^{2 m+8}+p^{2 m+9}-p^{3 m+1}-5 p^{3 m+2}-15 p^{3 m+3}-30 p^{3 m+4}-$ $45 p^{3 m+5}-51 p^{3 m+6}-45 p^{3 m+7}-30 p^{3 m+8}-15 p^{3 m+9}-5 p^{3 m+10}-p^{3 m+11}+4 p^{4 m+1}+$ $10 p^{4 m+2}+20 p^{4 m+3}+31 p^{4 m+4}+40 p^{4 m+5}+44 p^{4 m+6}+40 p^{4 m+7}+31 p^{4 m+8}+20 p^{4 m+9}+$ $\left.10 p^{4 m+10}+4 p^{4 m+11}+p^{4 m+12}\right)$, valid for $m \geq 1$.

We get $a_{5}^{\prime}(p)-a_{5}^{\prime}(1) a_{5}^{\prime}\left(p^{2}\right)=(p-1)\left((p-1) p\left(p^{2}+p+3\right)(p(p+2)+2)-10\right)$, which we see is positive, and thus we have proved the inequality (21) for $m=0$.

For $m \geq 1$, we get $a_{5}^{\prime}\left(p^{m+1}\right)^{2}-a_{5}^{\prime}\left(p^{m}\right) a_{5}\left(p^{m+2}\right)=\frac{(p-1)^{4} p^{3 m-13}}{p^{2}+p+1}\left(-p^{3 m}+p^{4 m}-p^{m+2}-\right.$ $4 p^{m+3}-10 p^{m+4}-16 p^{m+5}-19 p^{m+6}-16 p^{m+7}-10 p^{m+8}-4 p^{m+9}-p^{m+10}+2 p^{2 m+1}+$ $10 p^{2 m+2}+34 p^{2 m+3}+80 p^{2 m+4}+143 p^{2 m+5}+201 p^{2 m+6}+224 p^{2 m+7}+201 p^{2 m+8}+143 p^{2 m+9}+$ $80 p^{2 m+10}+34 p^{2 m+11}+10 p^{2 m+12}+2 p^{2 m+13}-8 p^{3 m+1}-32 p^{3 m+2}-88 p^{3 m+3}-188 p^{3 m+4}-$ $328 p^{3 m+5}-480 p^{3 m+6}-600 p^{3 m+7}-646 p^{3 m+8}-600 p^{3 m+9}-480 p^{3 m+10}-328 p^{3 m+11}-$ $188 p^{3 m+12}-88 p^{3 m+13}-32 p^{3 m+14}-8 p^{3 m+15}-p^{3 m+16}+6 p^{4 m+1}+23 p^{4 m+2}+64 p^{4 m+3}+$ $143 p^{4 m+4}+266 p^{4 m+5}+423 p^{4 m+6}+584 p^{4 m+7}+706 p^{4 m+8}+752 p^{4 m+9}+706 p^{4 m+10}+$ $584 p^{4 m+11}+423 p^{4 m+12}+266 p^{4 m+13}+143 p^{4 m+14}+64 p^{4 m+15}+23 p^{4 m+16}+6 p^{4 m+17}+$ $\left.p^{4 m+18}+p^{6}+2 p^{5}+p^{4}\right)$. The first factor is obviously positive for $p \geq 2$, and the second factor may be rearranged as $\left(752 p^{4 m+9}-646 p^{3 m+8}\right)+\left(706 p^{4 m+10}-600 p^{3 m+9}\right)+$ $\left(706 p^{4 m+8}-600 p^{3 m+7}\right)+\left(584 p^{4 m+11}-480 p^{3 m+10}\right)+\left(584 p^{4 m+7}-480 p^{3 m+6}\right)+\left(423 p^{4 m+12}-\right.$ $\left.328 p^{3 m+11}\right)+\left(423 p^{4 m+6}-328 p^{3 m+5}\right)+\left(266 p^{4 m+13}-188 p^{3 m+12}\right)+\left(266 p^{4 m+5}-188 p^{3 m+4}\right)+$ $\left(143 p^{4 m+14}-88 p^{3 m+13}\right)+\left(143 p^{4 m+4}-88 p^{3 m+3}\right)+\left(64 p^{4 m+15}-32 p^{3 m+14}\right)+\left(64 p^{4 m+3}-\right.$ $\left.32 p^{3 m+2}\right)+\left(23 p^{4 m+16}-8 p^{3 m+15}\right)+\left(23 p^{4 m+2}-8 p^{3 m+1}\right)+\left(6 p^{4 m+17}-p^{3 m+16}\right)+\left(6 p^{4 m+1}-\right.$ $\left.p^{3 m}\right)+\left(224 p^{2 m+7}-19 p^{m+6}\right)+\left(201 p^{2 m+8}-16 p^{m+7}\right)+\left(201 p^{2 m+6}-16 p^{m+5}\right)+\left(143 p^{2 m+9}-\right.$ $\left.10 p^{m+8}\right)+\left(143 p^{2 m+5}-10 p^{m+4}\right)+\left(80 p^{2 m+10}-4 p^{m+9}\right)+\left(80 p^{2 m+4}-4 p^{m+3}\right)+\left(34 p^{2 m+11}-\right.$ 
$\left.p^{m+10}\right)+\left(34 p^{2 m+3}-p^{m+2}\right)+10 p^{2 m+12}+10 p^{2 m+2}+2 p^{2 m+13}+2 p^{2 m+1}+2 p^{5}+p^{4 m+18}+$ $p^{4 m}+p^{6}+p^{4}$, where every term is positive for all $p \geq 2$, and we have thus proved the inequality (21) for $m \geq 1$. This concludes the proof of Proposition 24] for $n=5$.

The computations of $a_{5}^{\prime}\left(p^{m}\right)$ and $a_{5}^{\prime}\left(p^{m+1}\right)^{2}-a_{5}^{\prime}\left(p^{m}\right) a_{5}^{\prime}\left(p^{m+2}\right)$ may be verified with the Mathematica code provided at

http://www.math.kth.se/ holmin/files/x/a5prime_is_logconcave.

\section{Calculation of a measure}

In [Kat93] the asymptotics

$$
N_{n, 0}(T)=\frac{n-1}{\zeta(n)} w(B) T^{n(n-1)} \log T+O\left(T^{n(n-1)}\right)
$$

are given, where $B$ is the unit ball in $M_{n}(\mathbb{R})$. The measure $w$ on $M_{n}(\mathbb{R})$ is defined in [Kat93] as follows. Let $A_{u}:=\left\{A \in M_{n}(\mathbb{R}): A u=0\right\}$ be the space of matrices annihilating the nonzero vector $u \in \mathbb{R}^{n} \backslash\{0\}$. We define for (Lebesgue measurable) subsets $E \subseteq M_{n}(\mathbb{R})$ the measure $w_{u}(E):=\operatorname{vol}\left(E \cap A_{u}\right)$ where vol is the standard $n(n-1)$ dimensional volume on $A_{u}$, and define the measure $w(E):=(1 / 2) \int_{\mathrm{S}^{n-1}} w_{u}(E) d \nu(u)$, where $\nu$ is the standard Euclidean surface measure on the $(n-1)$-dimensional sphere $\mathrm{S}^{n-1}$.

We shall now calculate $w(B)$. The set $B \cap A_{u}$ is the unit ball in the $n(n-1)$-dimensional vector space $A_{u}$. Its volume does not depend on $u \neq 0$, and if $u=(0, \ldots, 0,1)$, then $B \cap A_{u}$ is the unit ball in $\mathbb{R}^{n(n-1)}$, when identifying $M_{n}(\mathbb{R})$ with $\mathbb{R}^{n^{2}}$. Denote by $V_{n(n-1)}$ the volume of the unit ball in $\mathbb{R}^{n(n-1)}$. Thus $w_{u}(B)=V_{n(n-1)}$, independently of $u \neq 0$, and

$$
w(B)=V_{n(n-1)} \frac{1}{2} \int_{\mathrm{S}^{n-1}} d \nu(u)=\frac{V_{n(n-1)} S_{n-1}}{2},
$$

where $S_{n-1}$ is the surface area of the sphere $\mathrm{S}^{n-1}$. The volume and surface area of the unit ball is well known, and we may explicitly calculate

$$
C_{0}:=w(B)=\frac{\pi^{n^{2} / 2}}{\Gamma\left(\frac{n}{2}\right) \Gamma\left(\frac{n(n-1)}{2}+1\right)} .
$$

Recalling from Theorem 6 the expression for $C_{1}$, we get the following relation.

$$
C_{1}=\frac{1}{\zeta(2) \cdots \zeta(n)} \frac{\pi^{n^{2} / 2}}{\Gamma\left(\frac{n}{2}\right) \Gamma\left(\frac{n(n-1)}{2}+1\right)}=\frac{1}{\zeta(2) \cdots \zeta(n)} C_{0} .
$$

\section{Acknowledgements}

I would like to thank my advisor Pär Kurlberg for suggesting this problem to me and for all his help and encouragement. 


\section{References}

[Coh93] Henri Cohen. A course in computational algebraic number theory, volume 138 of Graduate Texts in Mathematics. Springer-Verlag, Berlin, 1993.

[DRS93] W. Duke, Z. Rudnick, and P. Sarnak. Density of integer points on affine homogeneous varieties. Duke Math. J., 71(1):143-179, 1993.

[GN10] Alexander Gorodnik and Amos Nevo. The ergodic theory of lattice subgroups, volume 172 of Annals of Mathematics Studies. Princeton University Press, Princeton, NJ, 2010.

[Kat93] Y. R. Katznelson. Singular matrices and a uniform bound for congruence groups of $\mathrm{SL}_{n}(\mathbb{Z})$. Duke Math. J., 69(1):121-136, 1993.

[Men69] K. V. Menon. On the convolution of logarithmically concave sequences. Proc. Amer. Math. Soc., 23:439-441, 1969.

[Sie45] Carl Ludwig Siegel. A mean value theorem in geometry of numbers. Ann. of Math. (2), 46:340-347, 1945.

[Sta97] Richard P. Stanley. Enumerative combinatorics. Vol. 1, volume 49 of Cambridge Studies in Advanced Mathematics. Cambridge University Press, Cambridge, 1997.

[Wig05] Igor Wigman. Counting singular matrices with primitive row vectors. Monatsh. Math., 144(1):71-84, 2005. 